\title{
Assessment of Homogeneous and Heterogeneous Resonance Integral Tables and Their Applications to the Embedded Self-shielding Method
}

\author{
Yuxuan Liu and William R. Martin \\ Department of Nuclear Engineering and Radiological Sciences, University of Michigan \\ 2355 Bonisteel Blvd., Ann Arbor, MI, 48109-2104, USA
}

\author{
Corresponding Author: \\ Yuxuan Liu \\ yuxuanl@umich.edu
}

\begin{abstract}
Conventional resonance self-shielding methods are primarily based on equivalence theory, which allows the resonance integral (RI) table generated by homogeneous media to be useful for heterogeneous calculation. In the past two decades, a new RI table based directly on heterogeneous calculation has been developed and proved to be effective in the self-shielding calculation. This note compares the homogeneous and heterogeneous RI tables on the basis of derivation and numerical results. The limitations of the two RI tables and their applicability to the embedded self-shielding method (ESSM) are discussed.
\end{abstract}

\section{Keywords}

Resonance integral table; Homogeneous and heterogeneous table; ESSM

\section{Introduction}

Conventional resonance theory breaks the solution roadmap into two steps: (1) for homogeneous media, tabulate resonance integral (RI) of a resonance isotope against background cross section; (2) correlate heterogeneous system with homogeneous system using equivalence theory [1] so that the table generated for homogeneous media can be utilized for 
heterogeneous calculation. The equivalence relation between homogeneity and heterogeneity has been formulated and widely used in lattice calculation since 1960s until a recent heterogeneous formulation of equivalence theory being developed in the past 10-20 years [2-5]. The main difference of the heterogeneous formulation is that homogeneous slowing-down calculations against a range of background cross sections are replaced by a set of heterogeneous pin cell slowing-down calculations where background cross sections are implicitly varied by adjusting the heterogeneous pin configurations. Previous works independently claim that equivalence methods employing homogeneous and heterogeneous RI tables both produce satisfactory results for self-shielding calculation, but comparisons have rarely been performed to scrutinize the two types of tables. In addition, one is likely to be confused by the various approximations used in developing those tables, which however, seem all acceptable given the satisfactory results reported. The purpose of this note is to examine the two types of RI tables in the resolved resonance range for light water reactor (LWR) analysis. The theory of homogeneous RI table (Stamm'ler method [1]) is revisited and an alternative approach to understanding the heterogeneous RI table is provided. The dilemmas in generating and using the two types of RI tables are clarified. Applications of these RI tables to the embedded selfshielding method (ESSM) [6,7] are reinvestigated and the cause of the unsatisfactory results with ESSM reported by a recent paper [8] is discussed.

Section 2 reviews the derivation of homogeneous and heterogeneous RI tables. The approximations and their impacts on the accuracy of calculating effective cross section are discussed. To show the difference of two RI tables, the heterogeneous RI table is redeveloped by allowing the equivalence cross section as a function of continuous energy. Section 3 compares the homogeneous and heterogeneous RI tables within the framework of ESSM. Conclusions are drawn in Section 4.

\section{Homogeneous and Heterogeneous RI tables}

Resonance theory often starts with the neutron slowing-down equation in the resolved resonance energy range,

$$
\Omega \cdot \nabla \psi(r, \Omega, u)+\sum_{i s o} \Sigma_{t, i s o}(r, u) \psi(r, \Omega, u)=\frac{1}{4 \pi} \sum_{i s o} \int_{u-\varepsilon_{i s o}}^{u} \Sigma_{s, i s o}\left(r, u^{\prime}\right) \phi\left(r, u^{\prime}\right) \frac{e^{u^{\prime}-u}}{1-\alpha_{i s o}} d \dot{u}
$$

where $\psi(r, \Omega, u)$ and $\phi(r, u)$ are the neutron angular and scalar flux, respectively. $\Sigma_{x, i s o}$ is the macroscopic cross section of reaction channel $x$ for isotope iso. $\alpha_{i s o}=\left(\frac{A_{i s o}-1}{A_{i s o}+1}\right)^{2}$ and $\varepsilon_{i s o}=\ln \left(\frac{1}{\alpha_{i s o}}\right)$ are the maximum fraction of energy loss and maximum lethargy gain per neutron scattered off isotope iso, respectively. To achieve this equation, three assumptions regarding the source term has been made to the neutron transport equation: (1) direct fission source is neglected; (2) asymptotic scattering kernel is assumed so that upscattering is neglected; (3) the scattering source is treated by only considering isotropic elastic reactions in the laboratory system. For the fission spectrum of U-235, which is the primary isotope sustaining fission chain reaction for light water reactors, more than $99 \%$ of the direct fission neutrons are born in the fast energy region $\left(>10^{5} \mathrm{eV}\right)$. The neutron source in the slowing-down region is primarily provided by the scattered neutrons, so (1) is a good approximation. Assumption (2) is generally 
used in the conventional slowing-down calculations, but recent works $[9,10]$ have shown that the explicit treatment of resonance up-scattering for heavy nuclides in the epithermal energy range can increase the LWR Doppler coefficients by $10 \%$ relative to the asymptotic scattering kernel. In the current work, the asymptotic scattering kernel is still used for the slowing-down calculation. Although RI tables can be improved by including the upscattering treatment in solving the slowing-down equation, this is well outside of the comparison purpose of this work. For Assumption (3), a detailed explanation supporting it can be found in Ref. [11]. All the slowing-down calculations (including reference calculation) performed in this note are based on the foregoing assumptions, so RI table comparisons presented in the following content exclude errors caused by these assumptions.

This note primarily investigates variations of the Stamm'ler equivalence method. Other equivalence method is possible [12], but is not discussed in this note. Also, the Stamm'ler equivalence theory is limited to a single region of resonance materials without subdivision. This basic case is also chosen in evaluating and comparing various RI tables in this note. Advanced methods that handle multiple fuel regions can be found in Refs. $[13,14]$. In addition, the resonance interference is not discussed in this note since RI table is often generated for each resonance isotope independently. Interference effect can be accounted for by performing the slowing-down correction, as described in Ref. [14].

\subsection{Homogeneous RI table}

\subsubsection{Table generation with NR absorber and hydrogen}

For a homogeneous media, Equation (1) simplifies to

$$
\sum_{i s o} \Sigma_{t, i s o}(u) \phi(u)=\sum_{i s o} \int_{u-\varepsilon_{i s o}}^{u} \Sigma_{s, i s o}\left(u^{\prime}\right) \phi\left(u^{\prime}\right) \frac{e^{u^{\prime}-u}}{1-\alpha_{\text {iso }}} d u^{\prime}
$$

Homogeneous RI table is often generated with an approach similar to that in NJOY [15]. A homogeneous mixture of a resonance isotope and a background isotope is considered. Hydrogen is chosen to be the background isotope as all the resonances are narrow with respect to the energy loss of neutron scattering off hydrogen. This allows us to approximate the flux in the scattering integral of hydrogen as $1 / E$. Furthermore, assuming hydrogen is a pure scatterer with constant potential cross section $\Sigma_{p}$, the equation can be written as

$$
\left[\Sigma_{t, r e s}(u)+\Sigma_{p, H-1}\right] \phi(u)=\int_{u-\varepsilon_{\text {res }}}^{u} \Sigma_{s, r e s}\left(u^{\prime}\right) \phi\left(u^{\prime}\right) \frac{e^{u^{\prime}-u}}{1-\alpha_{\text {res }}} d u^{\prime}+\Sigma_{p, H-1}
$$

Divided by the number density of the resonance isotope, we obtain

$$
\left[\sigma_{t, r e s}(u)+\sigma_{0}\right] \phi(u)=\int_{u-\varepsilon_{\text {res }}}^{u} \sigma_{s, r e s}\left(u^{\prime}\right) \phi\left(u^{\prime}\right) \frac{e^{u^{\prime}-u}}{1-\alpha_{\text {res }}} d u^{\prime}+\sigma_{0}
$$

where $\sigma_{0}=N_{H-1} \sigma_{p, H-1} / N_{\text {res }}$. The solution $\phi(u)$ of Equation (4) is used to compute the effective cross section of group $g$ for reaction type $x$ 


$$
\sigma_{x, r e s, g}=\int_{\Delta u_{g}} \sigma_{x, r e s}(u) \phi(u) d u / \int_{\Delta u_{g}} \phi(u) d u
$$

By varying $N_{H-1}$ (and thus $\sigma_{0}$ from a few barns to the infinite dilution), a table of effective cross section versus background cross section $\sigma_{b}=\sigma_{0}$ can be obtained. To use this table for self-shielding calculation of a homogeneous mixture, the background cross section with NR approximation is computed as

$$
\sigma_{b}=\sum_{i s o \neq r e s} \Sigma_{p, i s o} / N_{r e s}
$$

Alternatively, some multigroup libraries tabulate the RI per lethargy group instead of effective cross section. By using NR approximation for all isotopes,

$$
\begin{gathered}
I_{x, \text { res }}=\frac{1}{\Delta u_{g}} \int_{u_{g-1}}^{u_{g}} \sigma_{x, \text { res }}(u) \phi(u) d u \approx \frac{1}{\Delta u_{g}} \int_{u_{g-1}}^{u_{g}} \sigma_{x, \text { res }}(u) \frac{\sigma_{p, \text { res }}+\sigma_{b}}{\sigma_{t, r e s}(u)+\sigma_{b}} d u=I_{x, \text { res }}\left(\sigma_{b}\right) \\
\bar{\phi}=\frac{1}{\Delta u_{g}} \int_{u_{g-1}}^{u_{g}} \phi(u) d u \approx \frac{1}{\Delta u_{g}} \int_{u_{g-1}}^{u_{g}} \frac{\sigma_{p, \text { res }}+\sigma_{b}}{\sigma_{t, r e s}(u)+\sigma_{b}} d u \\
=1-\frac{1}{\left(\sigma_{p, r e s}+\sigma_{b}\right) \Delta u_{g}} \int_{u_{g-1}}^{u_{g}} \sigma_{a, \text { res }}(u) \frac{\sigma_{p, r e s}+\sigma_{b}}{\sigma_{t, \text { res }}(u)+\sigma_{b}} d u=1-\frac{I_{a, r e s}\left(\sigma_{b}\right)}{\sigma_{p, r e s}+\sigma_{b}} \\
\sigma_{x}=\frac{I_{x, \text { res }}}{\bar{\phi}}=I_{x, \text { res }}\left(\sigma_{b}\right) /\left(1-\frac{I_{a, \text { res }}\left(\sigma_{b}\right)}{\sigma_{p, \text { res }}+\sigma_{b}}\right)
\end{gathered}
$$

Note the subscript of energy group is dropped for simplicity. Using Equation (7-3), RI and effective cross section can be converted back and forth, so the tabulation of RI or effective cross section is equivalent. Although NR is also applied to the resonance isotope in deriving Equation (7-3), the exact effective cross section from slowing-down calculation (Equation (4)) can be retrieved by the RI table, at least for the tabulated points. As to be seen later, this technique has been massively used in the resonance theory, i.e., computing the effective cross section using slowing-down calculation but formulating the relation between $\mathrm{RI}$ and background cross section with resonance approximations. This technique is also extended to develop the equivalence theory, whose consequences will be discussed later as well.

\subsubsection{Table generation with IR absorber and hydrogen}

Applying Equation (6) to isotopes other than hydrogen also assumes NR for these isotopes. This approximation is poor for the epithermal energy range where the resonance is relatively wide compared to the scattering energy loss against non-hydrogen isotopes. To improve the accuracy in the epithermal range, an intermediate resonance (IR) extension [16] is introduced for all isotopes

$$
\sum_{i s o} \Sigma_{t, i s o}(u) \phi(u)=\sum_{i s o} \lambda_{i s o} \Sigma_{p, i s o}+\sum_{i s o}\left(1-\lambda_{i s o}\right) \Sigma_{s, i s o}(u) \phi(u)
$$


Given that NR and Wide Resonance (WR) approximations are two limiting conditions of the resonance width relative to the energy loss per neutron scattering off an isotope, actual resonance width is assumed as a blend of the NR and WR that is modeled by the IR parameter $\lambda_{i s o}$. Rearranging the flux to the left hand side gives

$$
\phi(u)=\frac{\sigma_{b}}{\sigma_{a, r e s}(u)+\lambda_{r e s} \sigma_{R S, r e s}(u)+\sigma_{b}}
$$

where $\sigma_{b}=\frac{\sum_{i s o} \lambda_{i s o} \Sigma_{p, i s o}}{N_{\text {res }}}$ and $\sigma_{R S, \text { res }}(u)=\sigma_{s, \text { res }}(u)-\sigma_{p, \text { res }}(u)$. Similar to Equation

correlation between effective cross section and RI per lethargy group can be derived as

$$
\sigma_{x}=\frac{I_{x, r e s}}{\bar{\phi}}=I_{x, r e s}\left(\sigma_{b}\right) /\left(1-\frac{I_{a, r e s}\left(\sigma_{b}\right)+\lambda_{r e s} I_{R S, r e s}\left(\sigma_{b}\right)}{\sigma_{b}}\right)
$$

Using Equation (10), the homogeneous RI table with IR approximation can be obtained. It should be emphasized that effective cross sections $\left(\sigma_{a}, v \sigma_{f}, \sigma_{s}, \sigma_{R S}\right)$ are still collapsed using spectrum from Equation (4). However, the definition of background cross section in table generation is no longer $\sigma_{0}$ but is replaced by $\sigma_{b}=\lambda_{\text {res }} \sigma_{p, r e s}+\sigma_{0}$, given the mixture of resonance isotope and hydrogen. In addition, the RI table for resonance scattering $\sigma_{R S}$ is needed. By including the IR approximation for non-hydrogen scatterer, the accuracy of the RI table is improved near the epithermal energy range.

A further simplification of the IR approach is to eliminate the $\lambda_{\text {res }} I_{R S, \text { res }}\left(\sigma_{b}\right)$ term [1],

$$
\sigma_{x}=\frac{I_{x, r e s}}{\bar{\phi}}=I_{x, r e s}\left(\sigma_{b}\right) /\left(1-\frac{I_{a, r e s}\left(\sigma_{b}\right)}{\sigma_{b}}\right)
$$

As long as this term is eliminated consistently in table generation and utilization, the effective cross section can be retrieved at least for the tabulated points. In this case, the $I_{R S}$ table is no longer needed.

So far, three approaches to formulate homogeneous RI table have been discussed: NR, IR and IR without resonance scattering. They all solve the slowing-down equation for a mixture of resonance isotope and hydrogen, but the resonance approximations are varied in establishing the relation between $\mathrm{RI}$ and background cross section.

\subsubsection{Equivalence theory}

The application of homogeneous RI table to determine the RI for a heterogeneous application is based on equivalence theory. This usually starts with the collision probability form of Equation (1) [1] 


$$
V_{F} \Sigma_{t, F}(u) \phi_{F}(u)=V_{F} P_{F \rightarrow F}(u) Q_{F}(u)+\sum_{J \in M} V_{J} P_{J \rightarrow F}(u) Q_{J}(u)
$$

In this equation, $\boldsymbol{F}$ is denoted as fuel material and $\boldsymbol{M}$ denotes materials other than fuel (cladding, coolant, etc.). $V_{X}$ is the volume of region $X$. We assume a flat flux $\phi_{X}$ for fuel region that has a single mesh without subdivision. $P_{F \rightarrow F}(u)$ and $P_{J \rightarrow F}(u)$ are the first flight collision probabilities from fuel to fuel and from material $J$ to fuel. $Q_{X}(u)$ is the scattering source term for region $X$. In order to achieve an equivalence relation, the IR approximation is applied to the fuel region, while the NR approximation is applied to other regions. The latter rests on the fact that nuclides of non-fuel regions are light or intermediate, so the resonances are assumed to be narrow. Utilizing these approximations, the scattering source terms simplify to

$$
\begin{gathered}
Q_{F}(u)=\sum_{\text {iso }} \lambda_{\text {iso }} \Sigma_{p, F, \text { iso }}+\sum_{\text {iso }}\left(1-\lambda_{\text {iso }}\right) \Sigma_{s, F, i s o}(u) \phi_{F}(u)=\lambda_{F} \Sigma_{p, F}+\left(1-\lambda_{F}\right) \Sigma_{s, F}(u) \phi_{F}(u) \\
Q_{J}(u)=\Sigma_{p, J} \quad(J \in M)
\end{gathered}
$$

Note that the notation drops isotope index for brevity. Substitution of Equation (13) into Equation (12) for $Q_{F}(u)$ and $Q_{J}(u)$ yields

$$
V_{F} \Sigma_{t, F}(u) \phi_{F}(u)=V_{F} P_{F \rightarrow F}(u)\left[\lambda_{F} \Sigma_{p, F}+\left(1-\lambda_{F}\right) \Sigma_{s, F}(u) \phi_{F}(u)\right]+\sum_{J \in M} V_{J} P_{J \rightarrow F}(u) \Sigma_{p, J}
$$

Assuming $\Sigma_{t, J}(u)=\Sigma_{p, J}$ for the non-fuel materials and using the reciprocity relation $V_{X} P_{X \rightarrow Y}(u) \Sigma_{t, X}(u)=V_{Y} P_{Y \rightarrow X}(u) \Sigma_{t, Y}(u)$, Equation (14) can be transformed to

$$
\Sigma_{t, F}(u) \phi_{F}(u)=\left(1-P_{e s c}(u)\right)\left[\lambda_{F} \Sigma_{p, F}+\left(1-\lambda_{F}\right) \Sigma_{s, F}(u) \phi_{F}(u)\right]+\Sigma_{t, F}(u) P_{e s c}(u)
$$

where the escape probability is defined as $P_{e s c}(u)=\sum_{J \in M} P_{F \rightarrow J}(u)$. Physically, the escape probability is the first flight collision probability from the fuel region to the non-fuel regions. Various approximations have been developed for evaluating the escape probability [17-19] and the basic idea is to obtain an expression for the flux that gives an analytic form equivalent to the flux for a homogeneous system. Rational approximations have been proven effective to form this equivalence by introducing an escape cross section

$$
\Sigma_{e}=\frac{1}{l} \quad \text { where } l=\text { mean chord length of the fuel region }
$$

For a general case, the $\mathrm{N}$-term rational approximation to the fuel escape probability of an isolated pin with infinite coolant is given as

$$
P_{e s c}(u)=\sum_{n=1}^{N} b_{n} \frac{a_{n} \Sigma_{e}}{\sum_{t, F}(u)+a_{n} \Sigma_{e}} \quad\left(\sum_{n=1}^{N} b_{n}=1\right)
$$


Substitution of Equation (17) into Equation (15) gives the flux of the fuel region,

$$
\phi_{F}(u)=\frac{\sum_{n=1}^{N} b_{n} \frac{\lambda_{F} \Sigma_{p, F}+a_{n} \Sigma_{e}}{\sum_{t, F}(u)+a_{n} \Sigma_{e}}}{\sum_{n=1}^{N} b_{n} \frac{\sum_{t, F}(u)-\left(1-\lambda_{F}\right) \Sigma_{s, F}(u)+a_{n} \Sigma_{e}}{\sum_{t, F}(u)+a_{n} \Sigma_{e}}}
$$

However, an equivalent form to the homogeneous media can be achieved only for two cases [1]. One is applying NR to the fuel material $\left(\lambda_{F}=1\right)$ and then the flux becomes

$$
\phi_{F}(u)=\sum_{n=1}^{N} b_{n} \frac{\Sigma_{p, F}+a_{n} \Sigma_{e}}{\sum_{t, F}(u)+a_{n} \Sigma_{e}}=\sum_{n=1}^{N} b_{n} \frac{\sigma_{p, r e s}+\sigma_{b n}}{\sigma_{t, r e s}(u)+\sigma_{b n}}
$$

where $\sigma_{b n}=\frac{\sum_{i s o \neq r e s} \sum_{p, i s o}+a_{n} \Sigma_{e}}{N_{r e s}}$ and the summation is over isotopes in the fuel.

The second is to use the single-term rational approximation, which yields an equivalent flux resembling Equation (9)

$$
\phi_{F}(u)=\frac{\lambda_{F} \Sigma_{p, F}+\Sigma_{e}}{\Sigma_{a, F}(u)+\lambda_{F} \Sigma_{s, F}(u)+\Sigma_{e}}=\frac{\sigma_{b}}{\sigma_{a, r e s}(u)+\lambda_{r e s} \sigma_{R S, r e s}(u)+\sigma_{b}}
$$

where $\sigma_{b}=\frac{\sum_{i s o} \lambda_{i s o} \Sigma_{p, i s o}+\Sigma_{e}}{N_{\text {res }}}$.

Many lattice codes use Equation (19) rather than (20) since the single-term rational approximation cannot predict the fuel escape probability accurately over a range of optical lengths, even with the correction of Bell factor. Using Equation (19), the effective cross section of the fuel can be represented in a similar way as Equation (7)

$$
\begin{gathered}
I_{x, \text { res }}=\frac{1}{\Delta u_{g}} \int_{u_{g-1}}^{u_{g}} \sigma_{x, \text { res }}(u) \phi(u) d u \approx \sum_{n=1}^{N} \frac{b_{n}}{\Delta u_{g}} \int_{u_{g-1}}^{u_{g}} \frac{\sigma_{x, \text { res }}(u)\left[\sigma_{p, \text { res }}+\sigma_{b n}\right]}{\sigma_{t, \text { res }}(u)+\sigma_{b n}} d u=\sum_{n=1}^{N} b_{n} I_{x, \text { res }}\left(\sigma_{b n}\right) \\
\bar{\phi}=\frac{1}{\Delta u_{g}} \int_{u_{g-1}}^{u_{g}} \phi(u) d u \approx \sum_{n=1}^{N} \frac{b_{n}}{\Delta u_{g}} \int_{u_{g-1}}^{u_{g}} \frac{\sigma_{p, \text { res }}+\sigma_{b n}}{\sigma_{t, \text { res }}(u)+\sigma_{b n}} d u \\
=1-\sum_{n=1}^{N} \frac{b_{n}}{\left(\sigma_{p, r e s}+\sigma_{b n}\right) \Delta u_{g}} \int_{u_{g-1}}^{u_{g}} \sigma_{a, r e s}(u) \frac{\sigma_{p, \text { res }}+\sigma_{b n}}{\sigma_{t, \text { res }}(u)+\sigma_{b n}} d u=1-\sum_{n=1}^{N} b_{n} \frac{I_{a, \text { res }}\left(\sigma_{b n}\right)}{\sigma_{p, r e s}+\sigma_{b n}} \\
\sigma_{x}=\frac{I_{x, \text { res }}}{\bar{\phi}}=\sum_{n=1}^{N} b_{n} I_{x, \text { res }}\left(\sigma_{b n}\right) /\left(1-\sum_{n=1}^{N} b_{n} \frac{I_{a, \text { res }}\left(\sigma_{b n}\right)}{\sigma_{p, \text { res }}+\sigma_{b n}}\right)
\end{gathered}
$$


Equation (21-3) indicates that the effective cross section of a heterogeneous case can be calculated by a combination of homogeneous cases as long as the coefficients of rational approximation are properly determined. For a cylindrical geometry, Carlvik two-term expansion is utilized in many lattice codes.

To account for the inter-pin shielding effect for a fuel lattice, the Dancoff factor can be incorporated into the coefficients of the Carlvik two-term expansion [1]

$$
\begin{aligned}
& a_{1,2}=\frac{5 A+6 \mp \sqrt{A^{2}+36 A+36}}{2 A+2} \\
& b_{1}=\frac{\frac{4 A+6}{A+1}-a_{1}}{a_{2}-a_{1}}, \quad b_{2}=1-b_{1}
\end{aligned}
$$

where $A=D /(1-D)$ and $D$ is the Dancoff factor.

Since the multi-term rational approximation cannot be used with the IR approximation, in order to have the heterogeneous flux strongly resemble the homogeneous case, an empirical approach is used to replace the NR flux with the IR flux for each rational term in Equation (19) [1],

$$
\phi_{F}(u)=\sum_{n=1}^{N} b_{n} \frac{\sigma_{b n}}{\sigma_{a, r e s}(u)+\lambda_{r e s} \sigma_{R S, r e s}(u)+\sigma_{b n}}
$$

where $\sigma_{b n}=\frac{\sum_{i s o} \lambda_{i s o} \Sigma_{p, i s o}+a_{n} \Sigma_{e}}{N_{\text {res }}}$. Similar to Equation (21), the effective cross section using Equation (23) is then given as

$$
\sigma_{x}=\frac{I_{x, \text { res }}}{\bar{\phi}}=\sum_{n=1}^{N} b_{n} I_{x, \text { res }}\left(\sigma_{b n}\right) /\left(1-\sum_{n=1}^{N} b_{n} \frac{I_{a, r e s}\left(\sigma_{b n}\right)+\lambda_{\text {res }} I_{R S, r e s}\left(\sigma_{b n}\right)}{\sigma_{b n}}\right)
$$

This equation is used in conjunction with the RI table generated by Equation (10). Similarly, the $\lambda_{\text {res }} I_{R S, \text { res }}\left(\sigma_{b n}\right)$ term can be consistently eliminated in generation and use of the tables. So far, the fundamentals of generating homogeneous RI table and its applications to heterogeneous case have been discussed. Table 1 summarizes the taxonomy of homogeneous RI tables.

To compare the three homogeneous RI tables, a two-region isolated pin cell is tested. The radius of fuel region is $0.4 \mathrm{~cm}$, consisting of $\mathrm{U}-238$ and $0-16$ and is surrounded by infinite water. Carlvik two-term rational approximation is used in the equivalence relation. Using the energy structures of 172-group XMAS library [20] and 47-group CASL library [21], the three RI tables are generated with continuous-energy (CE) cross sections. These CE cross sections are obtained from the AMPX CE library [22], which was processed with ENDF/B-VII.0 [23]. Figures 1 and 2 compare the effective U-238 absorption using the three RI tables for the two group structures, 
respectively. The reference solution is obtained by solving the slowing-down equation with the same AMPX CE library.

The effective absorption for $\mathrm{U}-238$ resonance groups near the epithermal energy range is overestimated for both group structures, which is consistent with the results reported in Ref. [24]. Ref. [24] also proved that these errors are mainly due to the NR approximation utilized in the equivalence relation of Equation (19). In addition, our results in Figures 1 and 2 indicate that the replacement of NR flux by IR flux for each rational term in Equation (23) is unable to remove this error, though the effective cross sections are slightly better for some groups. Obviously, the empirical IR fix in Equation (23) is no longer exact in spite of retrieving the resonance scattering term. In the meantime, whether to include the resonance scattering term in Equation (23) is of little impact, which is also suggested by Figures 1 and 2. For further interest, Ref. [24] presented an approach to improve the accuracy of homogeneous RI table under the framework of equivalence theory.

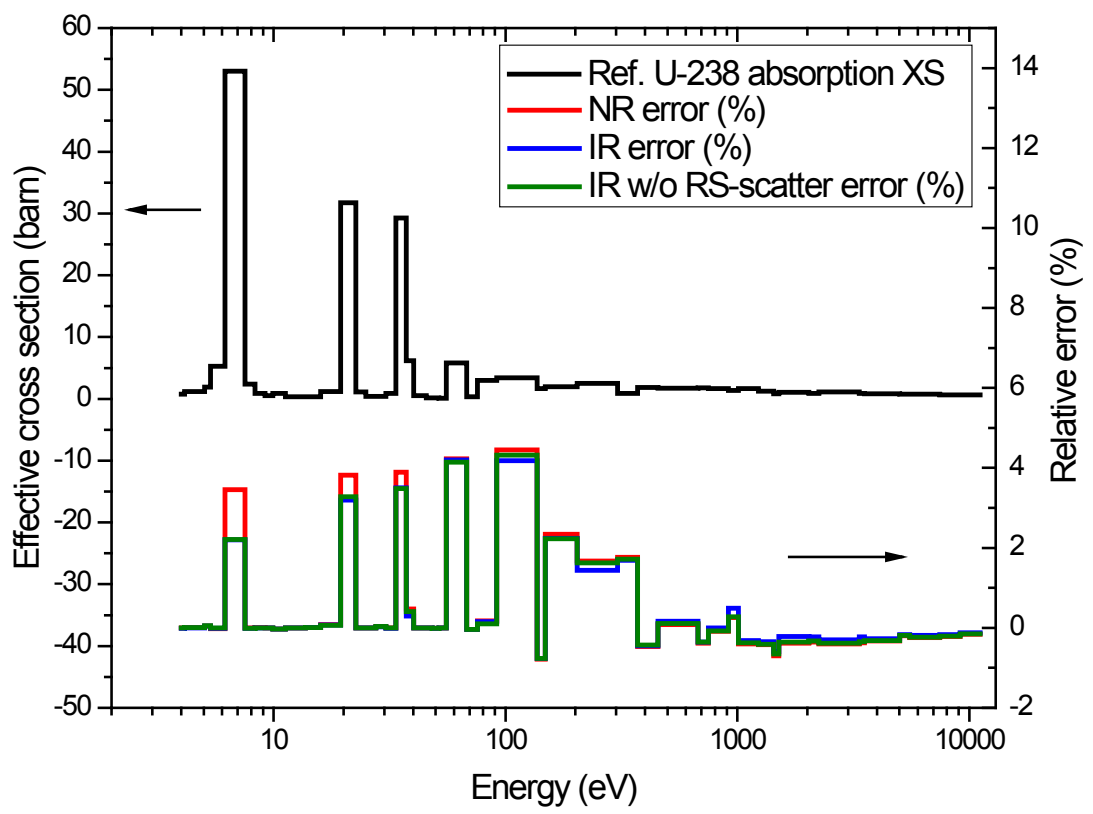

Figure 1 Comparison of homogeneous RI tables for an isolated pin in 172-group structure 


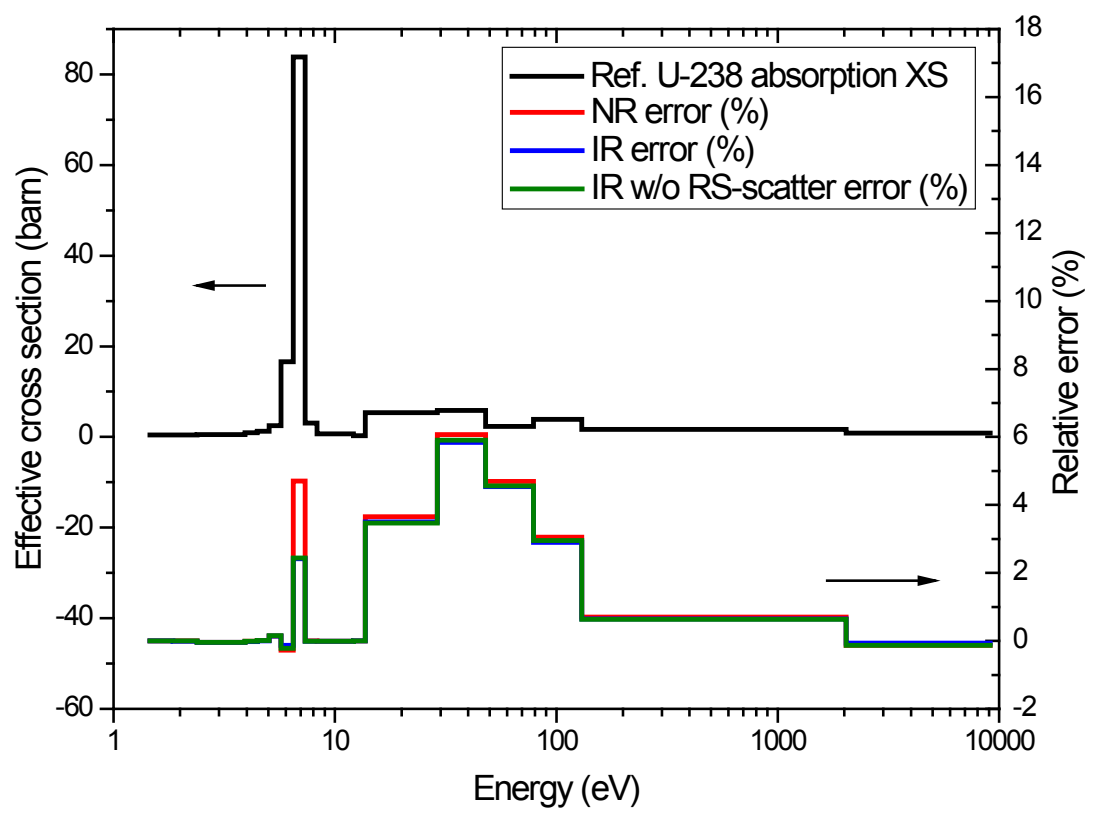

Figure 2 Comparison of homogeneous RI tables for an isolated pin in 47-group structure 
Table 1 Taxonomy of homogeneous RI tables

\begin{tabular}{|c|c|c|c|}
\hline $\begin{array}{c}\text { Resonance } \\
\text { Approximation }\end{array}$ & Generation & Use (homogeneous media) & Use (heterogeneous media) \\
\hline NR & $\begin{array}{l}\text { Slowing-down calculation: } \\
\qquad\left[\sigma_{t}(u)+\sigma_{0}\right] \phi(u)=\int_{u-\varepsilon}^{u} \sigma_{s}\left(u^{\prime}\right) \phi\left(u^{\prime}\right) \frac{e^{u^{\prime}-u}}{1-\alpha} d u^{\prime}+\sigma_{0} \\
\text { Tabulation: } \\
\qquad \sigma_{b}=\sigma_{0}, \quad I_{x}=\sigma_{x}\left(1-\frac{I_{a}}{\sigma_{p, r e s}+\sigma_{b}}\right)\end{array}$ & $\begin{array}{c}\sigma_{b}=\sum_{\text {iso } \neq \text { res }} \Sigma_{p, i s o} / N_{\text {res }} \\
\sigma_{x}=I_{x}\left(\sigma_{b}\right) /\left(1-\frac{I_{a}\left(\sigma_{b}\right)}{\sigma_{p, r e s}+\sigma_{b}}\right)\end{array}$ & $\begin{array}{c}\sigma_{b n}=\left(\sum_{i s o \neq r e s} \Sigma_{p, i s o}+a_{n} \Sigma_{e}\right) / N_{r e s} \\
\sigma_{x}=\frac{\sum_{n=1}^{N} b_{n} I_{x}\left(\sigma_{b n}\right)}{1-\sum_{n=1}^{N} b_{n} \frac{I_{a}\left(\sigma_{b n}\right)}{\sigma_{p, r e s}+\sigma_{b n}}}\end{array}$ \\
\hline IR & $\begin{array}{l}\text { Slowing-down calculation: } \\
\text { same as NR } \\
\text { Tabulation: } \\
\sigma_{b}=\sigma_{0}+\lambda \sigma_{p, r e s}, \quad I_{x}=\sigma_{x}\left(1-\frac{I_{a}+\lambda I_{R S}}{\sigma_{b}}\right)\end{array}$ & $\begin{array}{c}\sigma_{b}=\sum_{i s o} \lambda \Sigma_{p, i s o} / N_{\text {res }} \\
\sigma_{x}=I_{x}\left(\sigma_{b}\right) /\left(1-\frac{I_{a}\left(\sigma_{b}\right)+\lambda I_{R S}\left(\sigma_{b}\right)}{\sigma_{b}}\right.\end{array}$ & $\begin{array}{c}\sigma_{b n}=\left(\sum_{i s o} \lambda \Sigma_{p, i s o}+a_{n} \Sigma_{e}\right) / N_{r e s} \\
\sigma_{x}=\frac{\sum_{n=1}^{N} b_{n} I_{x}\left(\sigma_{b n}\right)}{1-\sum_{n=1}^{N} b_{n} \frac{I_{a}\left(\sigma_{b n}\right)+\lambda I_{R S}\left(\sigma_{b n}\right)}{\sigma_{b n}}}\end{array}$ \\
\hline $\begin{array}{l}\text { IR w/o RS- } \\
\text { scatter }\end{array}$ & $\begin{array}{l}\text { Slowing-down calculation: } \\
\qquad \text { same as NR } \\
\text { Tabulation: } \\
\qquad \sigma_{b}=\sigma_{0}+\lambda \sigma_{p, r e s}, \quad I_{x}=\sigma_{x}\left(1-\frac{I_{a}}{\sigma_{b}}\right)\end{array}$ & $\begin{array}{c}\sigma_{b}=\sum_{i s o} \lambda \Sigma_{p, i s o} / N_{r e s} \\
\sigma_{x}=I_{x}\left(\sigma_{b}\right) /\left(1-\frac{I_{a}\left(\sigma_{b}\right)}{\sigma_{b}}\right)\end{array}$ & $\begin{array}{c}\sigma_{b n}=\left(\sum_{i s o} \lambda \Sigma_{p, i s o}+a_{n} \Sigma_{e}\right) / N_{r e s} \\
\sigma_{x}=\frac{\sum_{n=1}^{N} b_{n} I_{x}\left(\sigma_{b n}\right)}{1-\sum_{n=1}^{N} b_{n} \frac{I_{a}\left(\sigma_{b n}\right)}{\sigma_{b n}}}\end{array}$ \\
\hline
\end{tabular}

The energy group subscript is dropped for simplicity 


\subsection{Heterogeneous RI table}

A new type of RI table based on heterogeneous formulation has been developed in the past 10-20 years. This approach claimed that a better consistency of the table generation and use could be achieved by solving the continuous-energy slowing-down equation over a set of heterogeneous pin cell configurations instead of homogeneous problems [2-5]. The theory underpinning this new kind of RI table is carefully redeveloped in this note to reveal the differences between homogeneous and heterogeneous tables.

As shown in the previous subsection, equivalence relation cannot be rigorously formulated by using IR approximation and multi-term rational approximation simultaneously. If Equation (20) is instead chosen to proceed (IR and single-term rational), large errors of effective cross sections are expected due to the error of single-term rational approximation unless the escape cross section is allowed to be a function of energy, i.e.,

$$
P_{e s c}(u)=\frac{\Sigma_{e q}(u)}{\Sigma_{t, F}(u)+\Sigma_{e q}(u)}
$$

Note for a fuel lattice the Dancoff effect can be absorbed into the equivalence cross section. Substitution of Equation (25) into Equation (15) gives

$$
\phi_{F}(u)=\frac{\lambda \sigma_{p}+\sigma_{e q}(u)}{\sigma_{a, r e s}(u)+\lambda_{\text {res }} \sigma_{R S, r e s}(u)+\lambda \sigma_{p}+\sigma_{e q}(u)}
$$

where $\lambda \sigma_{p}=\frac{\sum_{i s o} \lambda_{i s o} \Sigma_{p, i s o}}{N_{\text {res }}}$ and $\sigma_{e q}(u)=\frac{\Sigma_{e q}(u)}{N_{\text {res }}}$. The equivalence cross section $\Sigma_{e q}(u)$ is no longer a physical value like $\Sigma_{e}$, but is chosen to preserve the fuel escape probability at a specific energy for a certain geometry. If Equation (26) is integrated over an energy group,

$$
\phi_{F, g}=\frac{\left(\lambda \sigma_{p}+\bar{\sigma}_{e q, g}\right) \Delta u_{g}}{\sigma_{a, r e s, g}+\lambda_{r e s} \sigma_{R S, r e s, g}+\lambda \sigma_{p}+\sigma_{e q, g}}
$$

All cross sections with subscript $g$ are regular multigroup cross sections weighted by the neutron spectrum, except $\bar{\sigma}_{e q, g}$ which is the arithmetic mean within the energy group. Equation (27) suggests that the equivalence cross section can be calculated via the heterogeneous flux, however, we have two free variables $\bar{\sigma}_{e q, g}$ and $\sigma_{e q, g}$ in the equation. To simplify, the right hand side is forced to contain only one free variable in order to establish a one-on-one relation between flux and equivalence cross section

$$
\phi_{F, g}=\frac{\left(\lambda \sigma_{p}+\tilde{\sigma}_{e q, g}\right) \Delta u_{g}}{\sigma_{a, r e s, g}+\lambda_{r e s} \sigma_{R S, r e s, g}+\lambda \sigma_{p}+\tilde{\sigma}_{e q, g}}
$$

By defining the background cross section $\sigma_{b}=\lambda \sigma_{p}+\tilde{\sigma}_{e q, g}$, it can be expressed as 


$$
\sigma_{b, g}=\frac{\left(\sigma_{a, r e s, g}+\lambda_{r e s} \sigma_{R S, r e s, g}\right) \phi_{F, g}}{\Delta u_{g}-\phi_{F, g}}
$$

To evaluate $\sigma_{b, g}$ for a heterogeneous pin cell, the effective cross sections are first obtained by solving the slowing-down equation, Equation (1). Then a fixed source pin cell problem is solved consistently using IR approximation to obtain the heterogeneous flux $\phi_{F, g}$,

$$
\Omega \cdot \nabla \psi_{g}(r, \Omega)+\Sigma_{t, g}(r) \psi_{g}(r, \Omega)=\frac{1}{4 \pi}\left[(1-\lambda)\left(\Sigma_{R S, g}(r)+\Sigma_{p}(r)\right) \phi_{g}(r)+\lambda \Sigma_{p}(r) \Delta u_{g}\right]
$$

Note the effective cross sections in this equation come from the heterogeneous slowing-down calculation.

The relation of effective cross section (or RI) versus background cross section is established by solving Equations (1), (30) and (29). The same technique has been revisited, i.e., slowingdown equation is solved in calculating effective cross sections, while IR approximation is used to formulate the relation between $\mathrm{RI}$ and background cross section. To cover a range of background cross sections, a series of 1-D or 2-D heterogeneous pin cell problems with different geometrical factors and material compositions are considered. Table 2 presents a typical set of pin cell problems used for RI table generation in current transport codes such as HELIOS [4] and MPACT [21]. Configuration 5 is a typical PWR pin cell. Configurations 1-9 vary the moderator-tofuel ratio by changing moderator density or pitch. Configurations 10-16 gradually reduce the density of resonance isotope to further increase the background cross section. Since the equivalence cross section is forced to yield the correct fuel escape probability for a certain type of geometry (cylinder, slab, etc.), heterogeneous table can only be used for problems of that geometry.

Table 2 Heterogeneous pin cell configurations for RI table generation (typical heterogeneity)

\begin{tabular}{|c|c|c|c|c|c|c|c|c|}
\hline Configuration \# & $\mathbf{1}$ & $\mathbf{2}$ & $\mathbf{3}$ & $\mathbf{4}$ & $\mathbf{5}^{*}$ & $\mathbf{6}$ & $\mathbf{7}$ & $\mathbf{8}$ \\
\hline Ratio of pitch & 1.0 & 1.0 & 1.0 & 1.0 & 1.0 & 1.1 & 1.2 & 1.3 \\
\hline Ratio of Mod. density & 0.2 & 0.4 & 0.6 & 0.8 & 1.0 & 1.0 & 1.0 & 1.0 \\
\hline Ratio of Res. Iso. density & 1.0 & 1.0 & 1.0 & 1.0 & 1.0 & 1.0 & 1.0 & 1.0 \\
\hline Configuration \# & $\mathbf{9}$ & $\mathbf{1 0}$ & $\mathbf{1 1}$ & $\mathbf{1 2}$ & $\mathbf{1 3}$ & $\mathbf{1 4}$ & $\mathbf{1 5}$ & $\mathbf{1 6}$ \\
\hline Ratio of pitch & 1.4 & 1.4 & 1.4 & 1.4 & 1.4 & 1.4 & 1.4 & 1.4 \\
\hline Ratio of Mod. density & 1.0 & 1.0 & 1.0 & 1.0 & 1.0 & 1.0 & 1.0 & 1.0 \\
\hline Ratio of Res. Iso. density & 1.0 & 0.9 & 0.8 & 0.7 & 0.5 & 0.25 & 0.1 & 0.025 \\
\hline
\end{tabular}

${ }^{*}$ All the numbers are relative to configuration 5 :

Fuel radius $=0.4 \mathrm{~cm}$, pitch $=1.26 \mathrm{~cm}, \mathrm{~N}_{\mathrm{U}-238}=0.023 /($ barn $\cdot \mathrm{cm}), \mathrm{N}_{\mathrm{O}-16}=0.046 /(\mathrm{barn} \cdot \mathrm{cm})$, fuel temperature $=600 \mathrm{~K}$, and coolant $=600 \mathrm{~K}$ water 
As previously done, $\lambda \Sigma_{R S, g}$ term in Equations (29) and (30) can be eliminated in generating and using RI table simultaneously. A more aggressive approach in practice suggests moving the scalar flux term $\frac{1}{4 \pi}\left(\Sigma_{s, g}(r)+\lambda \Sigma_{p}(r)\right) \phi_{g}(r)$ to the left hand side and assuming an isotropic flux in the scattering term [3-5]

$$
\Omega \cdot \nabla \psi_{g}(r, \Omega)+\left[\Sigma_{a, g}(r)+\lambda \Sigma_{p}(r)\right] \psi_{g}(r, \Omega)=\frac{1}{4 \pi} \lambda \Sigma_{p}(r) \Delta u_{g}
$$

$\lambda \Sigma_{R S, g}$ should be consistently removed in calculating the background cross section

$$
\sigma_{b, g}=\frac{\sigma_{a, r e s, g} \phi_{F, g}}{\Delta u_{g}-\phi_{F, g}}
$$

The background cross section calculated via Equations (31) and (32) could be different from that via Equations (30) and (29). However, this simplification at least preserves the effective cross section of the tabulated points. Another reason in favor of Equation (31) is the faster calculation due to elimination of the scattering source iteration, which might not be a concern in generating the table, but is helpful to efficiently solve the fixed source problem when the table is in use. Table 3 summarizes the two heterogeneous RI tables.

Although the heterogeneous RI table can be used to generate subgroup parameters for the physical subgroup method $[3,4,5]$, a straightforward application of the heterogeneous RI table is ESSM. ESSM iteration is consistent with the generation of heterogeneous RI table $[6,7,14]$. An initial set of self-shielded cross sections can be obtained by table interpolation assuming $\tilde{\Sigma}_{e q}=0$. With the coefficients of these multigroup shielded cross sections, Equation (31) is solved for every 2-D plane and the resulting flux is used in Equation (32) to update the background cross sections. Then a new set of self-shielded cross sections can be obtained by RI table interpolation. The procedure iterates until the equivalence cross sections $\tilde{\Sigma}_{e q}$ of all resonant regions of the 2-D plane converge.

According to the pin configurations of Table 2, two heterogeneous RI tables (IR and IR w/o RS-scattering) are prepared. ESSM is performed to verify the isolated pin cell case as presented in the previous section. The results in Figures 3 and 4 indicate that simplifications made in Equations (31) and (32) do not undermine the accuracy of effective cross section for both group structures. In fact, the resonance scattering effect has been fully modeled by solving the slowing-down equation of heterogeneous pin cells. Equations (31) and (32) are only used to establish the correlation of $\mathrm{RI}$ and background cross section. The errors of $\sim 2 \%$ for major resonance groups in Figures 3 and 4 are due to other reasons discussed in the next section. 
Table 3 Taxonomy of heterogeneous RI tables

\begin{tabular}{|c|c|c|}
\hline $\begin{array}{c}\text { Resonance } \\
\text { Approximation }\end{array}$ & Generation & Use (heterogeneous media) \\
\hline IR & $\begin{array}{l}\text { Slowing-down calculation: } \\
\Omega \cdot \nabla \psi(r, \Omega, u)+\Sigma_{t}(r, u) \psi(r, \Omega, u)=\frac{1}{4 \pi} \sum_{i s o} \int_{u-\varepsilon_{i t o}}^{u} \sum_{s, i s o}\left(r, u^{\prime}\right) \phi\left(r, u^{\prime}\right) \frac{e^{u^{\prime}-u}}{1-\alpha_{i s o}} d \dot{u} \\
\text { Tabulation: } \\
\qquad \Omega \cdot \nabla \psi(r, \Omega)+\Sigma_{t}(r) \psi(r, \Omega)=\frac{1}{4 \pi}\left[(1-\lambda) \Sigma_{s}(r) \phi(r)+\lambda \Sigma_{p}(r) \Delta u\right] \\
\qquad \sigma_{b}=\frac{\left(\sigma_{a}+\lambda \sigma_{R S}\right) \phi}{\Delta u-\phi}, \quad I_{x}=\sigma_{x}\left(1-\frac{I_{a}+\lambda I_{R S}}{\sigma_{b}}\right)\end{array}$ & $\begin{array}{c}\text { Determine } \tilde{\Sigma}_{e q} \\
\sigma_{b}=\left(\sum_{i s o}^{N_{i s o}} \lambda \Sigma_{p, i s o}+\tilde{\Sigma}_{e q}\right) / N_{\text {res }} \\
\sigma_{x}=I_{x}\left(\sigma_{b}\right) /\left(1-\frac{I_{a}\left(\sigma_{b}\right)+\lambda I_{R S}\left(\sigma_{b}\right)}{\sigma_{b}}\right)\end{array}$ \\
\hline $\begin{array}{l}\text { IR w/o RS- } \\
\text { scatter }\end{array}$ & $\begin{array}{l}\text { Slowing-down calculation: } \\
\qquad \text { same as IR } \\
\text { Tabulation: } \\
\qquad \begin{aligned} \Omega \cdot \nabla \psi(r, \Omega)+\left[\Sigma_{a}(r)+\lambda \Sigma_{p}(r)\right] \psi(r, \Omega)=\frac{1}{4 \pi} \lambda \Sigma_{p}(r) \Delta u \\
\sigma_{b}=\frac{\sigma_{a} \phi}{\Delta u-\phi}, \quad I_{x}=\sigma_{x}\left(1-\frac{I_{a}}{\sigma_{b}}\right)\end{aligned}\end{array}$ & $\begin{array}{c}\text { Determine } \tilde{\Sigma}_{e q} \\
\sigma_{b}=\left(\sum_{i s o}^{N_{i s o}} \lambda \Sigma_{p, i s o}+\tilde{\Sigma}_{e q}\right) / N_{\text {res }} \\
\sigma_{x}=I_{x}\left(\sigma_{b}\right) /\left(1-\frac{I_{a}\left(\sigma_{b}\right)}{\sigma_{b}}\right)\end{array}$ \\
\hline
\end{tabular}

The energy group subscript is dropped for simplicity 


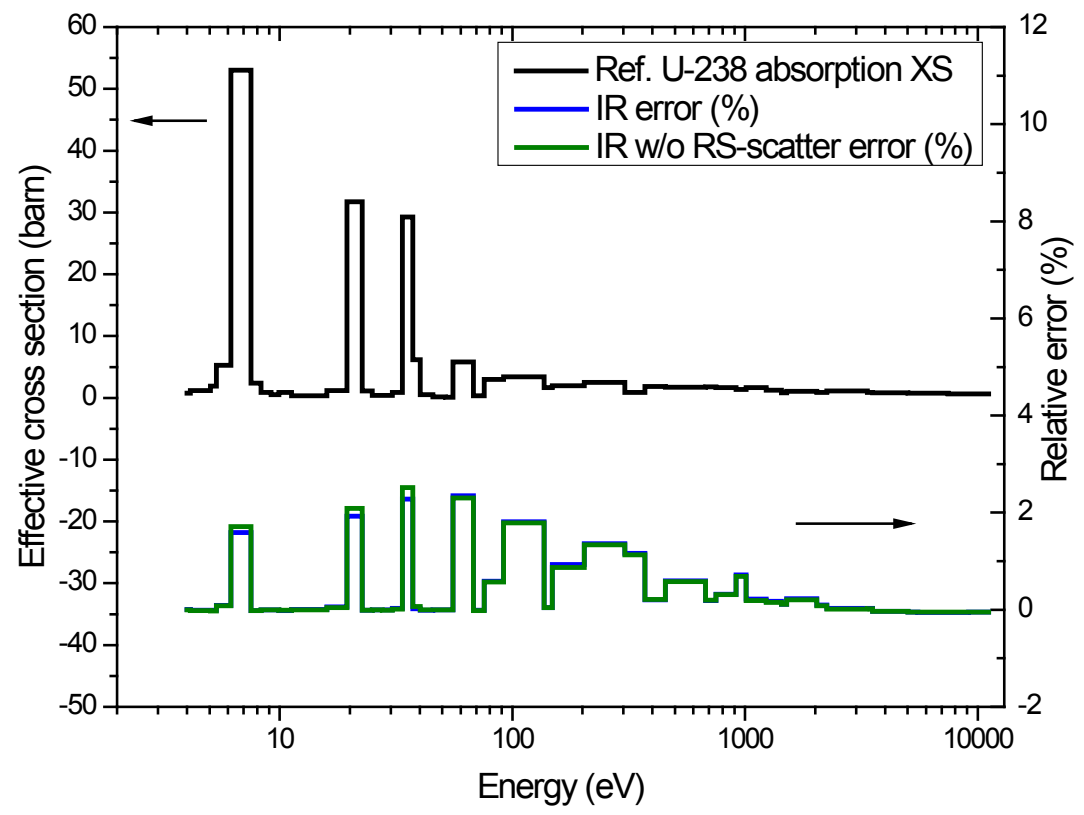

Figure 3 Comparison of heterogeneous RI tables for an isolated pin cell in 172-group structure

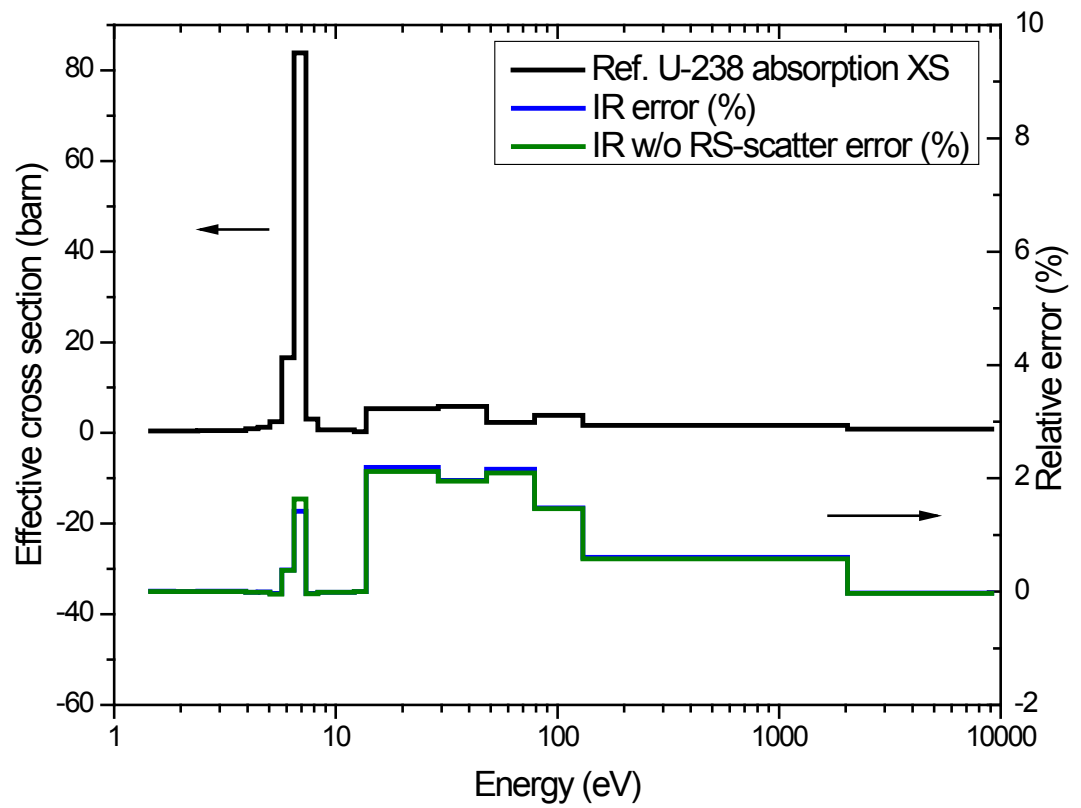

Figure 4 Comparison of heterogeneous RI tables for an isolated pin cell in 47-group structure

\section{Homogeneous and Heterogeneous RI tables for ESSM}

In the table generation step, homogeneous RI table only considers the volume effect, i.e., background cross section due to dilution of isotopes in the material volume. In comparison, heterogeneous RI table also includes the leakage effect by adding in the equivalence cross 
section. For different geometry types, the heterogeneous RI tables could be different since the equivalence cross section can only be forced to preserve the fuel escape probability of a certain geometry type. Also, homogeneous and heterogeneous RI tables should not be equivalent unless the volume and leakage portions of the background cross section have identical effects on the effective cross section. To investigate the difference between volume and leakage portions of the background cross section, another heterogeneous table is generated by pin configurations shown in Table 4. Compared to Table 2, oxygen is eliminated from the fuel material and the moderator density is extended to a large value. In this way, the volume effect is minimized and the majority of the background cross section is due to the equivalence cross section. By comparing these heterogeneous RI tables with the homogeneous RI table, the contribution of volume and leakage background to the effective cross section can be investigated. Note that all RI tables discussed in this section use IR approximation without resonance scattering term, which has been proved an adequate approach for both homogeneous and heterogeneous RI tables in Section 2.

Table 4 Heterogeneous pin cell configurations for RI table generation with leakage effect only (hyper heterogeneity)

\begin{tabular}{|c|c|c|c|c|c|c|c|c|}
\hline Configuration \# & $\mathbf{1}$ & $\mathbf{2}$ & $\mathbf{3}$ & $\mathbf{4}$ & $\mathbf{5 *}$ & $\mathbf{6}$ & $\mathbf{7}$ & $\mathbf{8}$ \\
\hline Ratio of pitch & 1.0 & 1.0 & 1.0 & 1.0 & 1.0 & 1.0 & 1.0 & 1.0 \\
\hline Ratio of Mod. density & 0.2 & 0.4 & 0.6 & 0.8 & 1.0 & 2.0 & 3.0 & 5.0 \\
\hline Ratio of Res. Iso. density & 1.0 & 1.0 & 1.0 & 1.0 & 1.0 & 1.0 & 1.0 & 1.0 \\
\hline Configuration \# & $\mathbf{9}$ & $\mathbf{1 0}$ & $\mathbf{1 1}$ & $\mathbf{1 2}$ & $\mathbf{1 3}$ & $\mathbf{1 4}$ & $\mathbf{1 5}$ & $\mathbf{1 6}$ \\
\hline Ratio of pitch & 1.0 & 1.0 & 1.0 & 1.0 & 1.0 & 1.0 & 1.0 & 1.0 \\
\hline Ratio of Mod. density & 20.0 & 20.0 & 20.0 & 20.0 & 20.0 & 20.0 & 20.0 & 20.0 \\
\hline Ratio of Res. Iso. density & 1.0 & 0.9 & 0.8 & 0.7 & 0.5 & 0.25 & 0.1 & 0.025 \\
\hline
\end{tabular}

*All the ratios are relative to configuration 5 :

Fuel radius $=0.4 \mathrm{~cm}$, pitch $=1.26 \mathrm{~cm}, \mathrm{~N}_{\mathrm{U}-238}=0.023 /(\mathrm{barn} \cdot \mathrm{cm}), \mathrm{N}_{\mathrm{O}-16}=0.0$

and coolant $=600 \mathrm{~K}$ water

We compare the U-238 effective absorption versus background cross section for the three RI tables, namely, homogeneous RI table (Homog RI), heterogeneous RI table using Table 2 (Typical-Heterog RI) and heterogeneous RI table using Table 4 (Hyper-Heterog RI). The results of Group 69 (55.60eV-67.90eV) in the 172-group structure and Group $19(6.47 \mathrm{eV}-7.34 \mathrm{eV})$ in the 47-group structure are shown in Figures 5 and 6, and the trends of Rl's are true for all other major resonance groups. As shown in the figures, Homog Rl gives the largest effective absorption, about $10 \%-15 \%$ more than that of Hyper-Heterog RI at $\sigma_{b}=50$ barn, a typical value in PWR applications. It is clear that the background cross section due to the volume effect yields larger effective cross section as compared with the same background cross section due to the leakage effect for cylindrical geometry. The values of Typical-Heterog RI are slightly greater than Hyper-Heterog RI, since the former includes oxygen in the fuel for table generation, which slightly enhances the volume effect. 


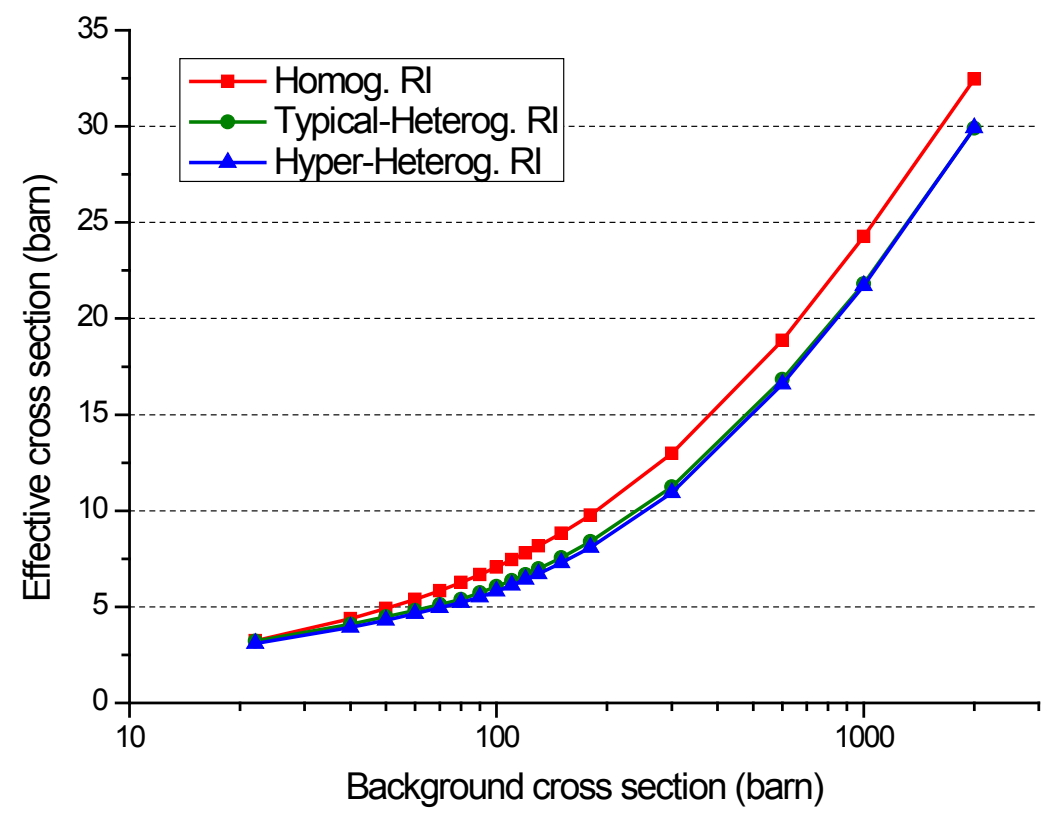

Figure 5 Group 69 (in 172-group structure) effective absorption of U-238 for three RI tables

To facilitate the comparison, effective cross sections of all tables are interpolated to the background cross sections for generating homogeneous RI table. The interpolation error is negligible due to dense background cross section points.

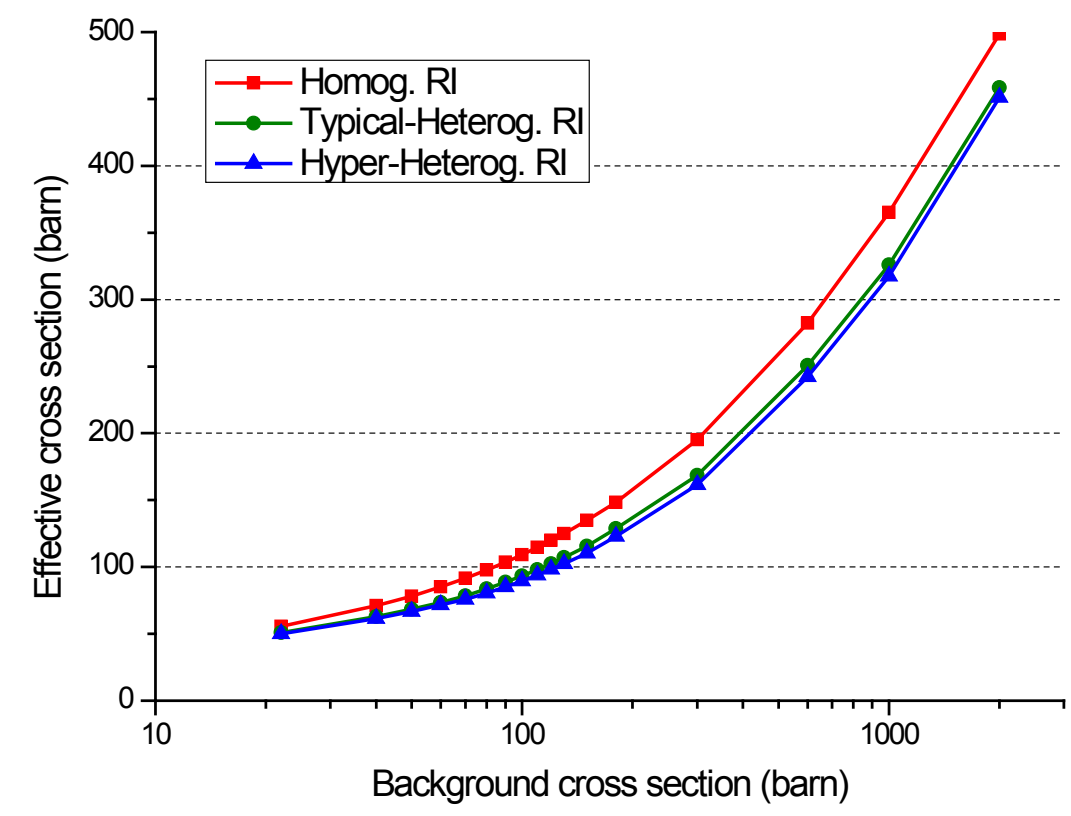

Figure 6 Group 19 (in 47-group structure) effective absorption of U-238 for three RI tables

Ideally, an additional parameter accounting for the ratio of the volume effect to the leakage effect should be included into heterogeneous RI table. Here we define this ratio 


$$
V / L=\lambda \tilde{\sigma}_{p} / \tilde{\sigma}_{e q, g}
$$

where $\lambda \tilde{\sigma}_{p}=\frac{\sum_{i s o \neq r e s} \lambda_{i s o} \Sigma_{p, i s o}}{N_{\text {res }}}$. Note the volume background does not include the resonance isotope itself because it would simply add a constant contribution in spite of $N_{\text {res }}$. Given a background cross section, previous discussion suggests a larger effective cross section for a larger $V / L$. In practice, however, this additional table dimension is often neglected (intentionally or unintentionally) by assuming Table 2 is able to cover the majority of applications. For example, in Configurations 1-9 of Table 2, variations are made on the water-touranium ratio by changing the fuel pitch or moderator density, but the volume background is fixed in a typical value of PWR fuel. In configurations 10-16, the density of the resonance isotope is then gradually reduced, but $V / L$ is kept constant.

In order to check the accuracy of current RI tables without the additional $V / L$ dimension, ESSM calculation is performed against two test cases. Test 1 is a fuel lattice case as shown in Figure 7. The boundary is reflective and all the pins are typical PWR fuel (U-238 and O-16, no U235 and no clad). Since water holes are included at the left corner, the effective cross section of $\mathrm{U}-238$ is rod-dependent. The effective absorption of Pins 2, 3 and 13 are compared with the reference slowing-down solution in Figure 8. As expected, Homog RI table overestimates the effective cross sections while Hyper-Heterog RI table underestimates them, which are consistent with the previous comparison of RI tables in this section. Since Homog RI table is used in Ref. [8] for ESSM, similar magnitude of error was identified. ESSM with Typical-Heterog table computes accurate effective cross section (error $<1 \%$ ) since $V / L$ ratios of the fuel pins in this case are well within those in the table generation. Also, the accuracy is similar for the two different energy group structures, indicating that the accuracy of heterogeneous table is not sensitive to the energy group structure.

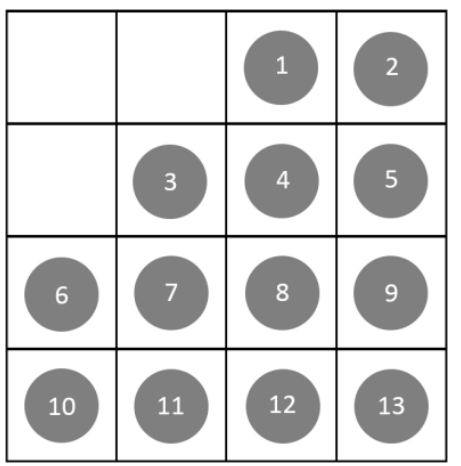

Figure 7 Fuel lattice layout of Test 1 
Pin 2 - 172G

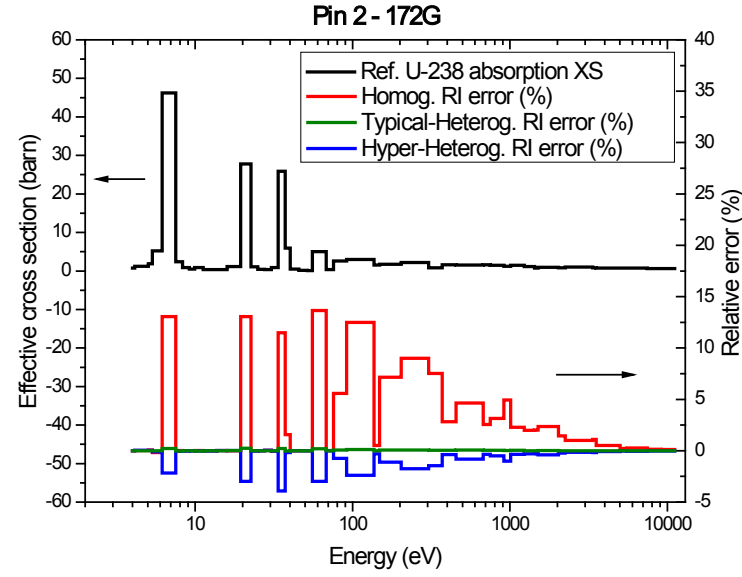

Pin 3-172G

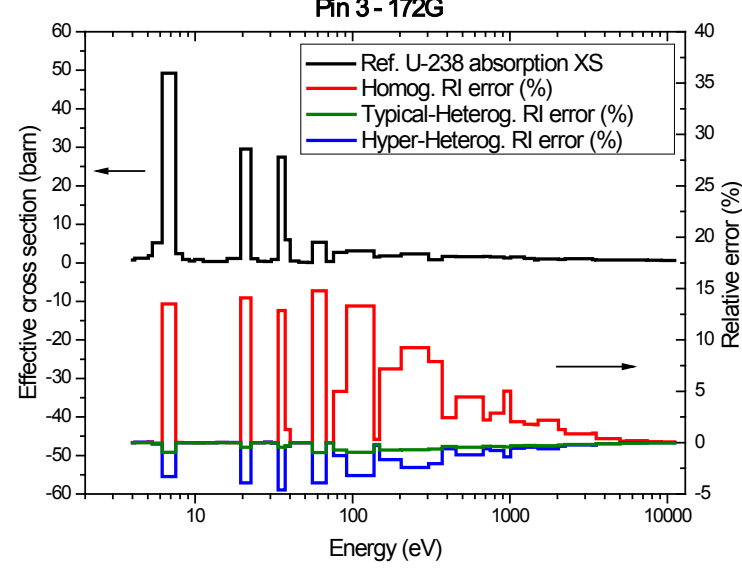

Pin $13-172 G$

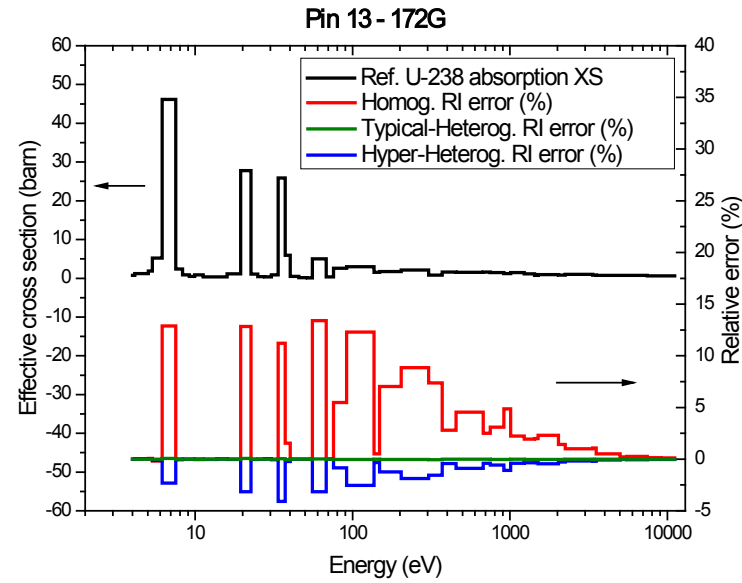

Pin 2-47G
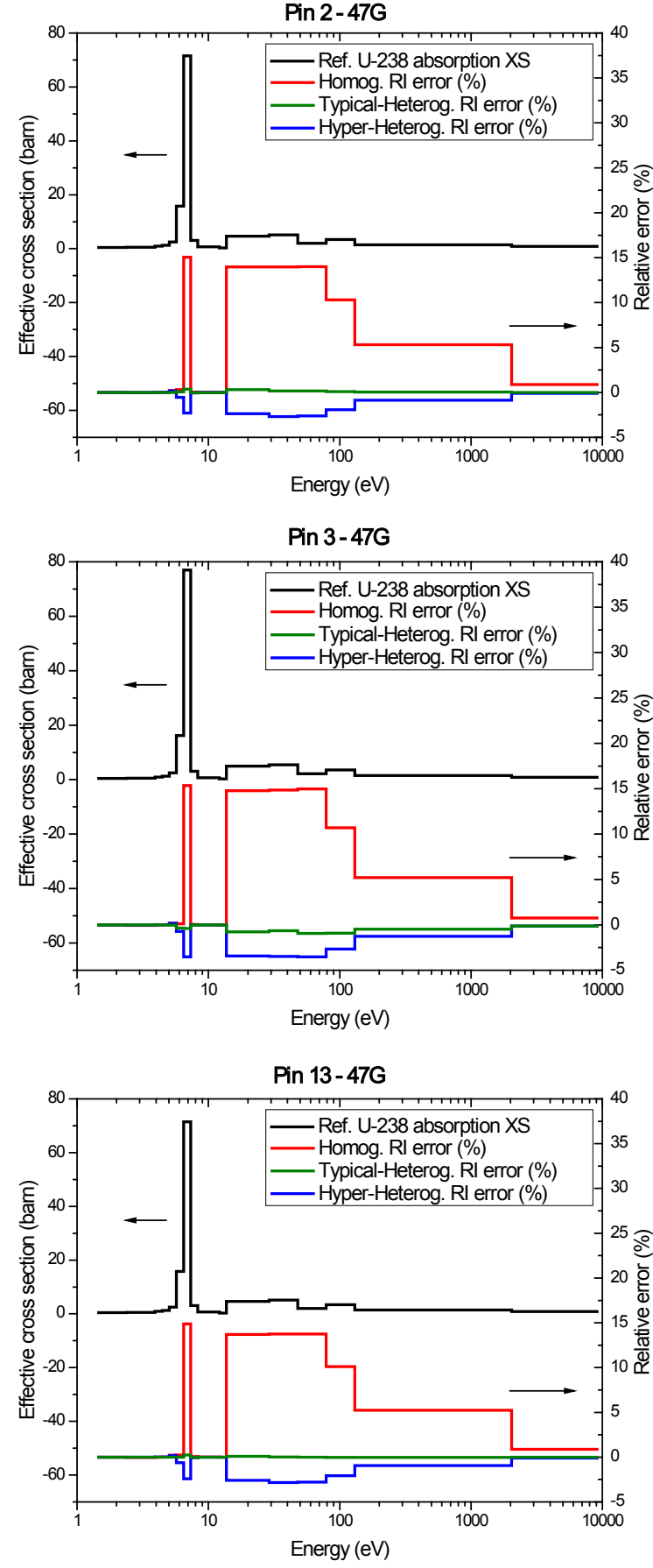

Figure 8 Assessment of RI tables for Test 1

Test 2 consists of three cases for which the $V / L$ ratios fall outside of the table generation (see Table 5). Cases 1 and 2 represent two limiting conditions where Case 1 homogenizes a typical pin cell, and Case 2 is an isolated fuel pin with infinite moderator. Case 3 reduces the U238 density to half of its typical value. Figure 9 compares the U-238 effective absorption for the three cases. ESSM with Typical-Heterog RI no longer computes correct effective cross section for 
Cases 1 and 2. For Case 3, the background cross section is almost doubled when the number density is reduced to half of the typical value. At this background cross section, the $V / L$ in generating Typical-Heterog RI table is smaller than that of Case 3. Consequently, negative errors in a magnitude of $2 \%-3 \%$ are seen for Case 3.

The two tests prove that Typical-Heterog RI table only produces accurate effective cross section if the $V / L$ of the problem is close to that of table generation. For a cross section library processed specifically for a certain reactor type (say PWR), mismatch of $V / L$ could happen when the resonance isotope density is significantly different from the value used in Typical-Heterog RI generation process. This may not be an issue for U-238 since its density does not vary too much with the fuel composition (enrichment, burnup, etc.). For other resonance isotopes such as U235 or plutonium where a typical density does not exist, $V / L$ may fall outside of the table generation, leading to a systematic bias of the effective cross section. Careful selection of the representative density for the table generation may minimize the bias for a specific range of applications, but cannot remove it. In practice, this bias is somewhat covered by the error due to crude resonance interference model, and thus is difficult to separate out. Nevertheless, consideration of $V / L$ as an additional argument for heterogeneous RI table would remove the bias and this is especially useful when one would like to extend the RI table to applications with various densities of minor resonance isotopes.

Table 5 Cases of test 2

\begin{tabular}{|c|c|c|cc|}
\hline \multirow{2}{*}{ Case } & \multicolumn{4}{|c|}{ Description } \\
\cline { 2 - 5 } & Pitch $(\mathrm{cm})$ & Fuel radius $(\mathrm{cm})$ & Fuel material (isotope number density) \\
\hline 1 & NA & NA & Homogenized from a typical pin cell ${ }^{(1)}$ \\
\hline 2 & 20 (infinite) & 0.4 & $\mathrm{~N}(\mathrm{U}-238)=0.023^{(2)}$ & $\mathrm{N}(\mathrm{O}-16)=0.0$ \\
\hline 3 & 1.26 & 0.4 & $\mathrm{~N}(\mathrm{U}-238)=0.0115$ & $\mathrm{~N}(\mathrm{O}-16)=0.046$ \\
\hline
\end{tabular}

(1) A typical pin cell: $0.4 \mathrm{~cm}$ fuel radius, $1.26 \mathrm{~cm}$ pitch, $\mathrm{N}(\mathrm{U}-238)=0.023$ and $\mathrm{N}(0-16)=0.046$

(2) Number density is in unit of $1 /($ barn $\cdot \mathrm{cm})$ 

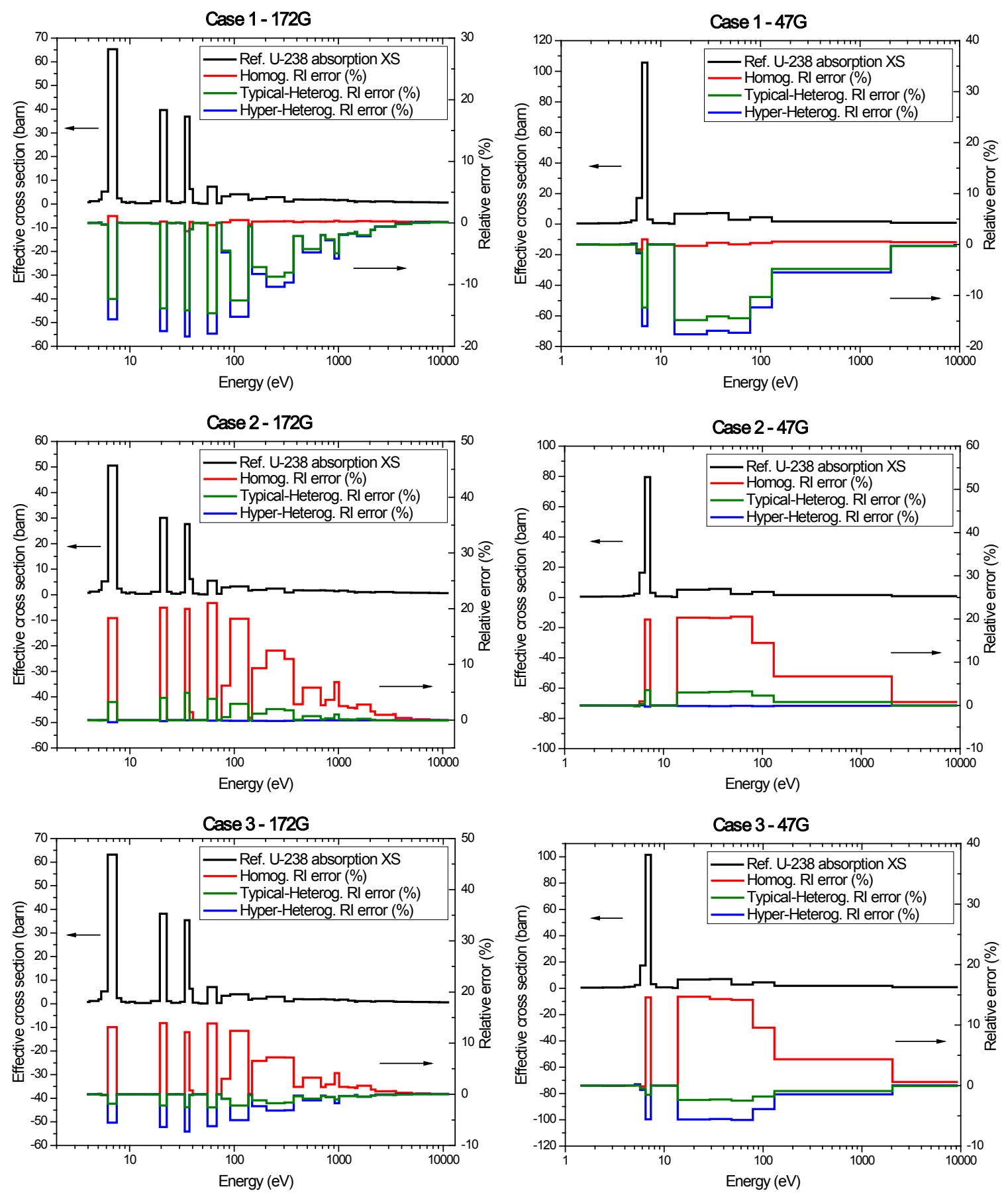

Figure 9 Assessment of RI tables for cases of Test 2

\section{Conclusion}

This note compares two leading equivalence methods used for the generation of RI tables, leading to the following conclusions: 
(1) Inaccuracy of homogeneous RI table for heterogeneous problem primarily results from the NR approximation utilized in deriving the equivalent heterogeneous flux. An empirical fix that replaces the NR flux by IR flux does not remove all the errors.

(2) Heterogeneous RI table retains the IR approximation by reducing to a single-term rational approximation, but the escape cross section has to be replaced by the forced equivalence cross section in order to preserve the fuel escape probability. Therefore, the heterogeneous RI table can only be used for problems in the same geometry type where the table is generated. In addition, consistently removing the resonance scattering term does not affect the accuracy of results.

(3) For the heterogeneous table, the background cross section due to the volume effect is worth more than that due to the leakage effect for heterogeneous RI table. The current generation approach is accurate as long as the $V / L$ of table generation is consistent with the problem of interest. An additional argument $V / L$ is needed for the heterogeneous RI table when one would like to extend the table to applications with various densities of minor resonance isotopes.

Further investigation on the $V / L$ ratio effect shall be performed in generation of the RI tables for minor resonance isotopes (other than U-238). For this purpose, the resonance interference should be properly modeled in order to reveal the error solely introduced by $V / L$ ratio.

\section{Acknowledgement}

This research was supported by the Consortium for Advanced Simulation of Light Water Reactors (http://www.casl.gov), an Energy Innovation Hub (http://www.energy.gov/hubs) for Modeling and Simulation of Nuclear Reactors under U.S. Department of Energy Contract No. DEAC05-00OR22725.

We would like to thank Dr. Kang-Seog Kim from Oak Ridge National Laboratory and Dr. Kord Smith from Massachusetts Institute of Technology for the invaluable discussions.

\section{Reference}

1. R. J. J. Stamm'ler and M. J. Abbate, Methods of Steady-state Reactor Physics in Nuclear Design, Academic Press, London (1983).

2. B. Fredin, U. Decher, A. Jonsson, A. Ferri and R. J. J. Stamm'ler, "Processing and Application of ENDF/B-VI in LWRs: Critical Experiments," Trans. Am. Nucl. Soc., 73, 419 (1995).

3. K. S. Kim, C. C. Lee, M. H. Chang and S. Q. Zee, "Monte Carlo Resonance Treatment for the Deterministic Transport Lattice Code," J. Korean Nucl. Soc., 35, 581 (2003).

4. R. J. J. Stamm'ler, et al., HELIOS Methods, Studsvik Scandpower (2003).

5. H. G. Joo, G. Y. Kim and L. Pogosbekyan, "Subgroup Weight Generation Based on Shielded 
Pin-cell Cross Section Conservation," Ann. Nucl. Energy, 36, 859 (2009).

6. S. G. Hong and K. S. Kim, "Iterative Resonance Self-Shielding Methods Using Resonance Integral Table in Heterogeneous Transport Lattice Calculations," Ann. Nucl. Eng., 38, 32 (2011).

7. M. L. Williams and K. S. Kim, "The Embedded Self-shielding Method," PHYSOR-2012, Knoxville, TN, April 15-20, (2012).

8. N. Gibson, K. Smith and B. Forget, "Simple Benchmark for Evaluating Self-shielding Models," Proc. M\&C-2015, Nashville, Tennessee, April 19-23 (2015).

9. D. Lee, K. Smith and J. Rhodes, "The Impact of ${ }^{238} \mathrm{U}$ Resonance Elastic Scattering Approximations on Thermal Reactor Doppler Reactivity," Ann. Nucl. Energy, 36, 274 (2009).

10. S. Z. Ghrayeb, A. M. Ougouag, M. Ouisloumen and K. N. Ivanov, "Multi-group Formulation of the Temperature-dependent Resonance Scattering model and its Impact on Reactor Core Parameters," Ann. Nucl. Energy, 63, 751 (2014).

11. N. Sugimura and A. Yamamoto, "Resonance Treatment Based on Ultra-fine-group Spectrum Calculation in the AEGIS Code," J. Nucl. Sci. Technol., 44, 958 (2007).

12. S. Loubiere, R. Sanchez, M.Coste, et al., APOLLO2 Twelve Years Later, Proc. M\&C-1999, Madrid, Spain (1999).

13. C. Stoker and Z. Weiss, "Spatially Dependent Resonance Cross Sections in a Fuel Rod," Ann. Nucl. Energy, 23, 765 (1996).

14. Y. Liu, W. Martin, M. Williams and K. Kim, "A Full Core Resonance Self-shielding Method Using a Continuous Energy Quasi-1D Slowing-down Solution that Accounts for Temperaturedependent Fuel Subregions and Resonance Interference," Nucl. Sci. Eng., 180, 247 (2015).

15. R. E. MacFarlane and D. W. Muir, "NJOY99.0 Code System for Producing Pointwise and Multigroup Neutron and Photon Cross Sections from ENDF/B Data," Report PSR480/NJOY99.0, Los Alamos National Laboratory, New Mexico (2000).

16. R. Goldstein and E. R. Cohen, "Theory of resonance absorption of neutrons," Nucl. Sci. Eng., 13, 132 (1962).

17. A. M. Weinberg and E. P. Wigner, The Physical Theory of Neutron Chain Reactors, University of Chicago Press, Chicago (1958).

18. G. I. Bell, "Theory of Effective Cross Sections," LA-2322, Los Alamos National Laboratory (1959).

19. I. Carlvik, "A Simplified Treatment of Spectrum Hardening in a Fuel Rod Caused by Selective Absorption," RFR-174, AB Atomenergi, Stockholm, Sweden (1962).

20. E. Sartori, "OECD/NEA Data Bank: Standard Energy Group Structures of Cross Section Libraries for Reactor Shielding, Reactor Cell and Fusion Neutronics Applications: VITAMIN-J, ECCO-33, ECCO-2000 and XMAS," JEF/DOC-315, Revision 3 - DRAFT (1990).

21. K. S. Kim, M. L. Williams, D. Wiarda and A. T. Godfrey, "Development of a New 47-Group Library for the CASL Neutronics Simulators," Proc. M\&C-2015, Nashville, Tennessee, April 19-23 (2015).

22. M. E. Dunn and N. M. Greene, "AMPX-2000: A Cross-Section Processing System for 
Generating Nuclear Data for Criticality Safety Applications," Trans. Am. Nucl. Soc., 86, 118 (2002).

23. M. B. Chadwick, et al., "ENDF/B-VII.0: Next Generation Evaluated Nuclear Data Library for Nuclear Science and Technology," Nucl. Data Sheets, 107, 2931 (2006).

24. Q. Zhang, H. Wu, L. Cao and Y. Zheng, "An Improved Resonance Self-Shielding Calculation Method Based on Equivalence Theory," Nucl. Sci. Eng., 179, 233 